\title{
Mid-infrared interferometry with high spectral resolution
}

Edward H. Wishnow, William Mallard, Vikram Ravi, Sean Lockwood, Walt Fitelson, et al.

Edward H. Wishnow, William Mallard, Vikram Ravi, Sean Lockwood, Walt Fitelson, Dan Wertheimer, Charles H. Townes, "Mid-infrared interferometry with high spectral resolution," Proc. SPIE 7734, Optical and Infrared Interferometry II, 773409 (21 July 2010); doi: 10.1117/12.857656

Event: SPIE Astronomical Telescopes + Instrumentation, 2010, San Diego, California, United States 


\title{
Mid-infrared interferometry with high spectral resolution
}

\author{
Edward H. Wishnow, William Mallard, Vikram Ravi, Sean Lockwood, Walt Fitelson, \\ Dan Werthimer, Charles H. Townes \\ Space Sciences Laboratory, University of California, Berkeley CA, USA 94720-7450
}

\begin{abstract}
The Infrared Spatial Interferometer (ISI) is a three telescope interferometer system that operates near 11 microns wavelength using heterodyne detection with $\mathrm{CO}_{2}$ lasers as local oscillators. Stellar measurements have been made using consistent instrumentation for 20 years, allowing comparisons of stellar sizes of red giant and Mira stars over time intervals which are long in comparison to stellar luminosity periods. Recent visibility and closure phase measurements of the star Betelgeuse have been fitted to simple image models and these results have been added to the 17 year record of stellar observations. A new area of investigation of stellar properties at very high spectral resolution will begin in the 2010-2011 observing season. The design of a new digital spectrometer-correlator system is discussed. This system will obtain visibility measurements on-and-off individual spectral lines and the continuum, simultaneously.
\end{abstract}

Keywords: stellar interferometry, interferometry instrumentation, mid-infrared astronomy

\section{INTRODUCTION}

The ISI is a three telescope interferometer located at the Mt. Wilson Observatory. The telescopes have a Pfund optical design with $1.65 \mathrm{~m}$ diameter primary mirrors mounted in movable semi-trailers. This design has a number of advantages: it is low to the ground and quite stable, a large primary can be fitted within a semi-trailer envelope, and the optical bench at the focus of the telescope does not change orientation with telescope pointing. The disadvantage is that only about half the sky can be observed from a N-S orientation of the optical axis. Fringes were first obtained with a two telescope system in 1988 and the third telescope was added in 2001. The telescopes can be arranged over various configurations and at present they form a nearly equilateral triangle with $\sim 36 \mathrm{~m}$ baselines. This configuration yields interferometric fringes with peak separations of 0.16 " to 0.06 ", permitting resolution of stellar sources, or features, of about half this size.

The ISI makes both frequent and long term observations of red giant and Mira stars providing a measurement record of stellar sizes, shapes and asymmetries, and the changes of these quantities over time. These stars are often surrounded by dust and they may be obscured at visible wavelengths. Mid-IR radiation from the star $(\sim 11 \mu \mathrm{m}$ wavelength) penetrates the dust better than shorter wavelengths and mid-IR radiation is also emitted by the warm dust. ISI observations in this band therefore give information about both the star and the surrounding dust. The ISI has high spectral resolution that allows avoidance of strong spectral lines due to gases surrounding these stars and therefore stellar continuum sizes can be observed without confusion due to molecular gases. A new spectrometer-correlator system is being built that will enable simultaneous observations of stars on-and-off individual spectral lines in order to trace the distribution of gases in extended stellar atmospheres. This paper discusses recent measurements of stellar changes with time, the motivation and design of the new digital correlator system, and some details regarding the correlator data analysis.

\subsection{Heterodyne detection}

The ISI is a unique optical-IR interferometer as it uses heterodyne detection in a manner similar to a radio telescope interferometer. The ISI operates near 11 microns wavelength $(27 \mathrm{THz})$ with $\mathrm{CO}_{2}$ lasers as local oscillators.

The two telescope detection scheme has been previously reported by Hale et al., ${ }^{1}$ and Figure 1 shows the current ISI detection scheme. Infrared radiation from a star is focused by a telescope onto a HgCdTe mixer. Radiation from the local oscillator (LO) is also focused onto the mixer and the resulting heterodyne signal, the intermediate frequency (IF), has a bandwidth of $+/-2.7 \mathrm{GHz}$. Each LO is set to a frequency, offset slightly from the master laser oscillator by phase

Optical and Infrared Interferometry II, edited by William C. Danchi, Françoise Delplancke, Jayadev K. Rajagopal, Proc. of SPIE Vol. 7734, 773409 - (c) 2010 SPIE · CCC code: 0277-786X/10/\$18 · doi: 10.1117/12.857656 
locking the beat frequency between the two lasers to a synthesized frequency (F1, F2 and F3 in Figure 1). The error signal from the phase lock loop (PLL) drives a piezo-actuated mirror to slightly change the laser cavity length and thereby shift the LO frequency. The synthesized frequency is varied to account for the "natural fringe frequency" associated with the interferometer's motion as the Earth rotates. The synthesized frequency is injected into each telescope's phase lock loop so that when the telescope IF signals are brought together and multiplied, the correlated signals are modulated at fixed fringe frequencies of 86,107 and $193 \mathrm{~Hz}$ for the telescope baseline pairs 1-2, 2-3 and 3-1, respectively. An analog-to-digital converter (ADC) records the data at $500 \mathrm{~Hz}$.

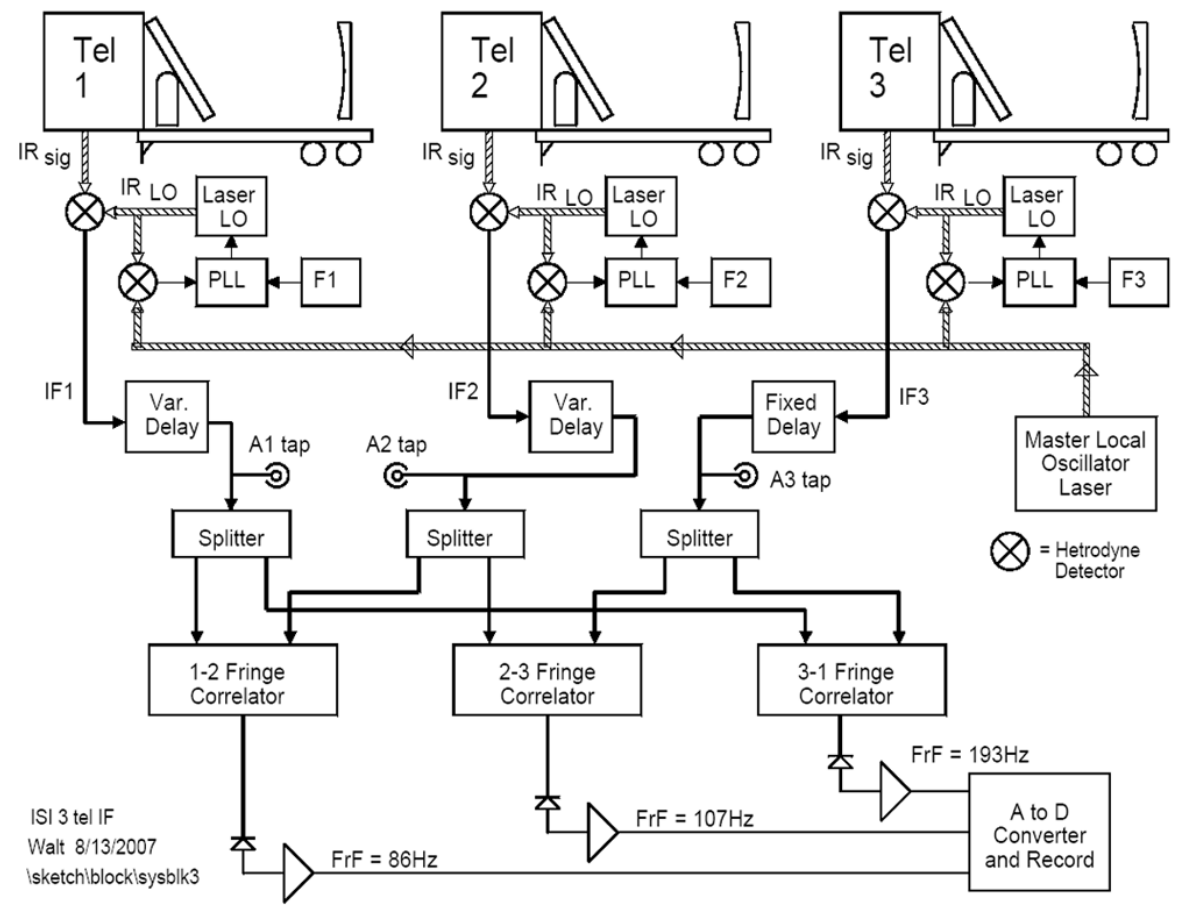

Figure 1. ISI system diagram. Radiation from a star is combined with that from $\mathrm{CO}_{2}$ laser local oscillators. The resulting IF signals are delayed, correlated and low-pass filtered. The interference fringes occur at frequencies chosen to be 86,107 and $193 \mathrm{~Hz}$. The delay lines automatically compensate for differential geometric and signal paths through the telescopes to the correlators with the required accuracy of $0.5 \mathrm{~cm}$, which is determined by the bandwidth used. Signals are sent to the new spectrometer/correlator at taps A1, A2, A3.

The radio frequency $(\mathrm{RF})$ power of the interference fringe is divided by the product of the total RF power independently received by each telescope to obtain the visibility at a particular $U V$ Fourier spatial frequency. A range of spatial frequencies are sampled by the variations in apparent baselines due to the rotation of the Earth and a broader range of spatial frequencies can be obtained by moving the telescopes. The heterodyne process at each telescope detects only the plane wave components of the stellar radiation. When brought together these signals interfere completely, whereas wavefronts distorted by atmospheric disturbances will not interfere. As a result, the measured visibility has some immunity to atmospheric fluctuations, though phase delays due to the atmosphere are still present.

\section{TEMPORAL VARIATIONS OF STARS}

The ISI has previously conducted detailed measurements of the star Mira over the course of a stellar luminosity cycle. ${ }^{2,3}$ Recently, the asymptotic giant branch (AGB) star W Hydrae has been observed and visibility variations over intermediate spatial frequencies are shown in the left panel of Figure 2. ${ }^{4}$ The measurements were conducted (from top to bottom) in Feb. 2008, June 2008, and Jan. 2009, and the luminosity phases are noted on the figures. The figures show visibilities due primarily to a dust shell; the data does not extend to high enough spatial frequencies to fully resolve the star which is assumed to have an angular radius of 25 mas. The abscissae are in spatial frequency units (SFU) or $10^{5}$ cyc/rad, where for comparison, $1 \mathrm{SFU} \sim 0.5 \mathrm{cyc} / \mathrm{arcsec}$. The data has been fitted with curves which are the sum of two uniform disk (UD) functions: one for the star and another for the dust shell. Temporal variations in the visibility arise 
from the outward expansion of this shell of material. The increase in the radius of this shell, from 47 to 67 mas over the course of a year, indicates a velocity of $9 \mathrm{~km} / \mathrm{sec}$, given a distance to the star of $104 \mathrm{pc}$.

The top right panel shows data from the ISI over the period 1994-2009. Although the dust shell varies over 2008, the entire data set is fairly consistent and it is combined to determine the radial distribution of material around the star. The visibility data is fitted with a polynomial function plus a Gaussian function, where the Gaussian part accounts for dust surrounding this star at fairly large angular scales. The Fourier transform of the fitted visibility, extended to high spatial frequencies using a 25 mas UD for the star, is shown in the lower right panel. This one-dimensional integrated intensity profile shows that W Hydrae consists of a central star surrounded by material just beyond the stellar radius, and a second shell of material at a radius of approximately 100-200 mas. The mid-IR observations are consistent with diameters of 36 to 62 mas which have been measured over the wavelength range $1-4 \mu \mathrm{m} .{ }^{5}$ Radio observations at $7 \mathrm{~mm}$ wavelength show a 69x46 mas elliptical shape for the star and a $\mathrm{SiO}$ shell of maser emission at a radius of $\sim 50$ mas. ${ }^{6}$ Observations at $1.3 \mathrm{~cm}$ wavelength show $\mathrm{H}_{2} \mathrm{O}$ maser emission at a radius of $\sim 100$ mas. ${ }^{7}$ The sizes of these two maser emission regions correspond generally to the shells observed here.
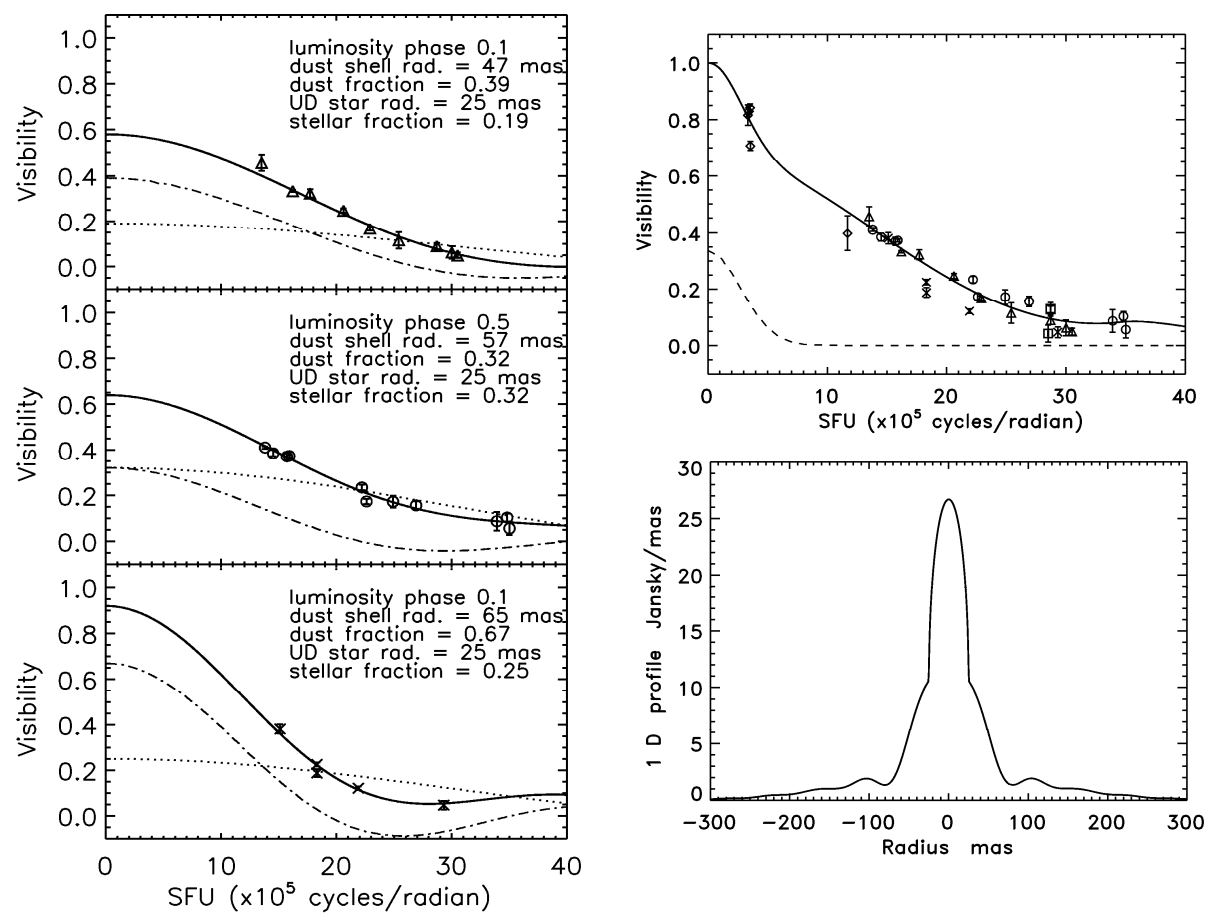

Figure 2. Left panel, Visibility measurements of W Hydrae over three observing periods covering about a year. The visibility is fitted with a model (solid line) which is the sum of two uniform disk curves representing the star (dotted line) and a dust shell (dot-dashed line). Absscissae are in spatial frequency units (SFU) of $10^{5}$ cyc/rad. Upper right panel, ISI visibility data over the time period 1994-2009. The data has been fitted with a polynomial plus a Gaussian denoted by the solid curve (the Gaussian part is shown by the dashed line). Lower right panel, the one-dimensional intensity distribution of W Hydrae obtained from the fit shown in the upper right panel.

The red supergiant star Betelgeuse was observed during Nov. 2009, shortly after the Station Fire threatened the observatories on Mt. Wilson. This data is shown in Figure 3 where the upper left panel shows the visibility data from the three ISI baselines. The lower left panel shows the closure phase measurements plotted as a function of the telescope 2-3 position angle. While mid-IR fringe amplitudes are measurable despite atmospheric distortions, the fringe phases are not. The closure phase, however, is measurable and it gives information on the source symmetry, i.e. the closure phase is non-zero for an intensity distribution which is not point-inversion symmetric. With only three telescopes, the ISI does not sample enough of the $U V$ plane to unambiguously form images, so the two-dimensional visibility data and closure phases are fitted to simple source distribution models. The upper right panel shows the best fit of a uniform disk plus a point source, where this probably is the simplest model that has a non-zero closure phase. The stellar radius is $25.83+/-0.2$ mas, the stellar intensity is $55 \%$ of the total 11 micron flux, and the point source has $1.2 \%$ of the total flux 
(the remainder of the flux is due to a $\sim 1$ arcsec dust shell not observed at high spatial frequencies). The fact that the point source lies outside the disk indicates that the model is not completely realistic. The fit is performed on the visibility data, but for comparison, points denoting UD radii for each visibility measurement are plotted (the colors indicate baselines 1-2, red; $2-3$, green; 3-1, blue). These colored points are only for purposes of guiding the interpretation of the fits. The lower right panel is the best fit uniform ellipse plus a point source. In this case, the semi-major and semiminor axes are: 25.6 and 27.4 mas, respectively, and the stellar and point source flux fractions are comparable to the UD plus point source model. Both models have nearly the same reduced chi-squared value and a reasonable interpretation is that the source is slightly brighter in the east and slightly north direction. While a simple uniform ellipse will fit the visibility data, it does not account for the non-zero closure phase. This data has been analyzed using methods developed during previous studies of Betelgeuse in 2006 where the star showed a brightening to the south with about $5 \%$ of the total mid-IR flux. ${ }^{8}$
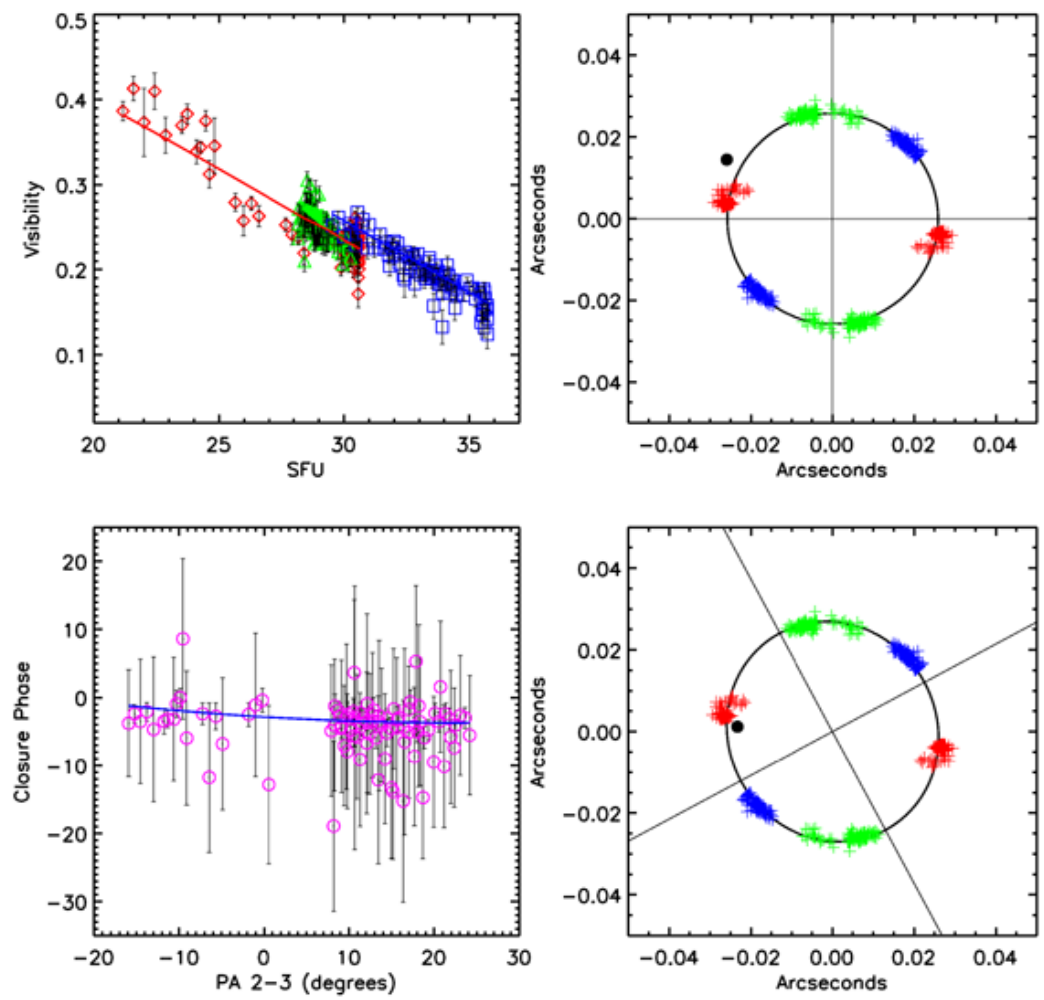

Figure 3. ISI measurements of Betelgeuse in Nov. 2009. Upper left panel, visibility measurements vs. spatial frequency (in SFU). Measurements from the telescope 1-2 baseline are indicated with red diamonds, the 2-3 baseline are indicated with green triangles, and the 3-1 baseline are shown with blue squares. Lower left panel, closure phases vs. the position angle of the telescope 2-3 baseline. The upper right panel shows the best fit UD+point source model; the lower right panel shows the best fit uniform ellipse+point source model. The reduced chi-squared for the models is nearly equal indicating that the true stellar image is brighter to the east and slightly north (in these figures North is at the top and East is to the left).

The ISI also has been performing long-term monitoring of red giant stars and these measurements show rather interesting changes in stars and their environs over decade time scales. For example, the visibility of the red supergiant star $\alpha$ Herculis over intermediate spatial frequency scales was observed to change rather dramatically over the period 1990--1993 and since 1998, the star has had a visibility near unity. This behavior may be interpreted as the formation and subsequent dissipation of dust surrounding the star.

A recent examination of the long-term record of Betelgeuse showed a diminishing of this star's size by about $15 \%$ over 16 years. ${ }^{10}$ The visible photometric record does show variations in the stars brightness of about 0.5 magnitudes, but these variations are not correlated with the apparent size. The uniform disk size determined from the Nov. 2009 data has been added to this record and this is shown below as the red diamond in Figure 4. The recent increase in apparent size is a change in the overall trend and validation of this result is underway. Red supergiant stars such as Betelgeuse vary in 
brightness and size variations are to be expected, though not previously measured. The variation in Figure 4 may be associated with the evolution of large-scale convection cells in the stellar atmosphere. Numerical models of red giant stars also indicate changes on various time scales. ${ }^{11}$ This ISI long-term record of stellar size variations provides a new observable for stellar theory and modeling.

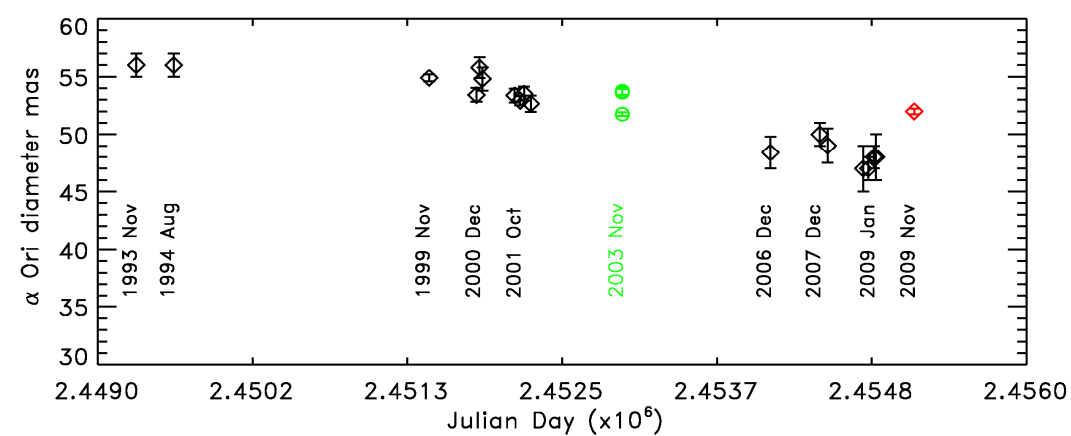

Figure 4. The diameter of Betelgeuse as a function of time. Black points denote ISI measurements at $11.15 \mathrm{~mm}$. Green points show measurements made by Perrin et. al at the VLT at 10.03 (lower), 11.04 (upper), 11.15 (upper) mm. ${ }^{11}$ The red point shows an average of recent Nov. 2009 ISI measurements; it is consistent with the UD+point disk in Figure 3.

\section{STELLAR SPECTROSCOPIC INTERFEROMETRY}

Red giant stars are very large and they have outer atmospheres composed of low density gas and dust. As a result these stars do not have a well defined surface, as distinguished from the appearance of the sun when observed in the visible. Diameter measurements of these stars vary by as much as a factor of two over the near-IR band, and Betelgeuse has an apparent diameter which varies by $27 \%$ over the mid-IR. ${ }^{12}$ This apparent size variation with wavelength is due to changes in the stellar opacity that are determined by a number of factors. Major contributors to the opacity are spectral features of simple molecules such as $\mathrm{CO}, \mathrm{OH}$ and $\mathrm{H}_{2} \mathrm{O}$ that surround these stars. The molecules form in either an extended stellar atmosphere, or in a separate molecular layer which lies at some distance from the star; this molecular layer model has been called a MOL-Sphere. ${ }^{13}$ The nature of the stellar opacity and the underlying contributors to it, the abundance, temperature and distribution of molecules in the stellar environment, are all issues that are not well understood and need more observational data.

In order to observe the presence and distribution of these molecules, a new spectrometer-correlator system is being developed. The ISI presently has a rather high spectral resolution of 5000 (double sideband) and the new instrument will have a resolution of over 500000 (although both sidebands will overlap). The new instrument will enable visibility measurements on-and-off individual molecular spectral lines simultaneously. Other stellar interferometers have also developed spectroscopic-interferometry systems, but they have generally been of moderate spectral resolution emphasizing molecular band observations. For example, the VLT-AMBER operates in the near-IR with a resolution of $12000,{ }^{14}$ and the Keck interferometer and VLT-MIDI systems operate in the mid-IR with resolutions of about 20 and 200 , respectively. The ISI will have a unique very high resolution capability in the mid-IR.

The new system is predicated on previous ISI spectroscopic measurements which were conducted by Monnier et al. ${ }^{15}$ and Weiner et. al. ${ }^{2,3}$ Monnier et al. observed the formation of ammonia $\left(\mathrm{NH}_{3}\right)$ and silane $\left(\mathrm{SiH}_{3}\right)$ at distances of 20 and 80 stellar radii, respectively, rather far from the central source of IRC +10216 . This work used an analog filterbank spectrometer on a two telescope interferometer. Visibility measurements of Mira conducted by Weiner et al. are shown in Figure 5, and this figure illustrates the potential capabilities of the new ISI system. The right panel shows visibility measurements in spectral regions with, and without, a water line. The left panels show the stellar spectrum over the continuum and water line regions. The smooth curves are measurements obtained using the TEXES mid-IR high resolution spectrometer ${ }^{16}$ and the "stick spectra" are predicted intensities of $\mathrm{H}_{2} \mathrm{O}$ lines at $1750 \mathrm{~K}$ from the HITRAN/HITEMP database. ${ }^{17}$ The ISI detection bands are shown with dashed lines where the apparent shifts of the bands are due to the barycentric motion of the Earth. Notice that the water line near $902 \mathrm{~cm}^{-1}$ has an "inverse P-Cygni lineshape" indicating gas flowing towards, and away, from the star. It is possible that a spectroscopic-interferometer system could locate these regions of gas motions in relation to the star. 

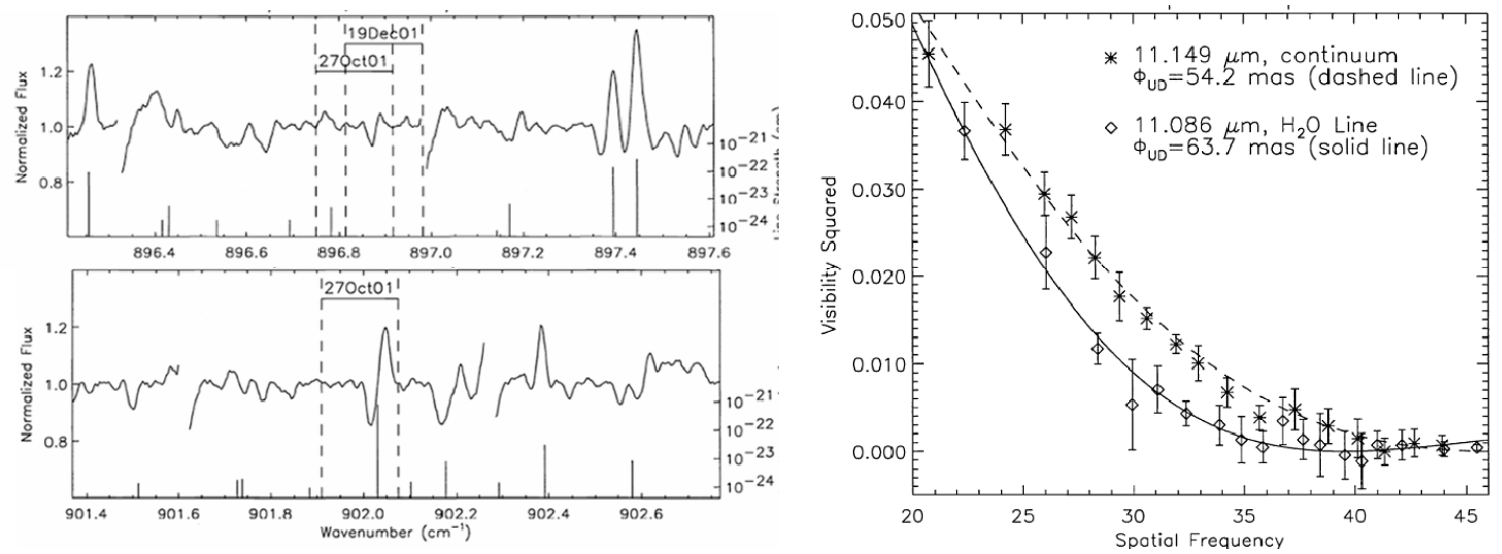

Figure 5. Mid-IR spectra and visibility measurements of Mira. Upper left panel shows the continuum region and the lower left panel shows the $\mathrm{H}_{2} \mathrm{O}$ line region $\left(11.149 \mu \mathrm{m}=896.94 \mathrm{~cm}^{-1}, 11.086 \mu \mathrm{m}=902.04 \mathrm{~cm}^{-1}\right)$, dashed lines indicate ISI detection bands. Right panel: visibility squared of Mira showing a larger size measured over the $\mathrm{H}_{2} \mathrm{O}$ line region. From Weiner et al. ${ }^{2}$

The new correlator system will be used initially for visibility measurements of red giant and AGB stars in spectral regions containing $\mathrm{H}_{2} \mathrm{O}$ and $\mathrm{OH}$ lines. $\mathrm{H}_{2} \mathrm{O}, \mathrm{OH}$ lines and continuum regions of interest are selected by predicting the molecular spectrum based on stellar atmospheric temperatures and the recessional velocity of the star. The region of interest must then fall within $+/-2.7 \mathrm{GHz}$ of a $\mathrm{CO}_{2}$ laser line which will then be used as the local oscillator. Figure 6 shows a relatively transparent region of the Earth's atmosphere and a set of laser lines for two isotopologues of $\mathrm{CO}_{2}$ chosen to avoid absorption lines of telluric ${ }^{16} \mathrm{O}^{12} \mathrm{C}^{16} \mathrm{O}$. The "stick spectra" show the intensities of $\mathrm{OH}$ (black) and $\mathrm{H}_{2} \mathrm{O}$ (green) spectral lines which might be expected in an extended stellar atmosphere.

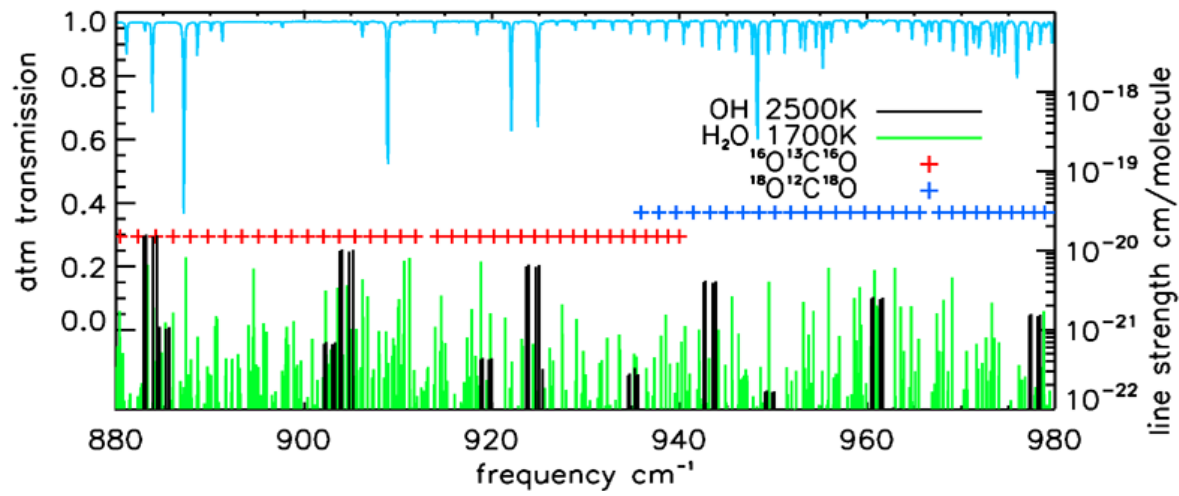

Figure 6. Atmospheric transmission vs. frequency (upper blue curve). Predicted intensities of $\mathrm{OH}$ and $\mathrm{H}_{2} \mathrm{O}$ lines are shown as "stick spectra"; laser line frequencies are indicated by red and blue + symbols.

The new correlator system is designed to have a spectral resolution matched to the expected stellar linewidths. The narrowest features will be thermally broadened, and $\mathrm{H}_{2} \mathrm{O}$ at $1000 \mathrm{~K}$ has a Doppler width of $0.0024 \mathrm{~cm}^{-1}$ (HWHM) or $\sim 72 \mathrm{MHz}$. The spectral resolution of $\sim 47 \mathrm{MHz}$ per channel is designed to slightly oversample the Doppler width and to give a convenient 64 channels across a $3 \mathrm{GHz}$ RF band. The turbulent flows of gases in the stellar atmosphere may further broaden or shift spectral lines, and when appropriate, spectral channels will be averaged together.

\subsection{Spectrometer-correlator system design}

The new ISI correlator is based on high-speed digital processing of the RF signals from the heterodyne detection system. It is being built in collaboration with the Center for Astronomy Signal Processing and Electronics Research (CASPER) at UC Berkeley, which develops open-source tools to build radio astronomy instrumentation. ${ }^{18}$ The correlator runs on the CASPER-developed ROACH board (Reconfigurable Open Architecture Computing Hardware) which uses a Xilinx Virtex 5 FPGA (Field Programmable Gate Array). It is an FX architecture correlator, which means that the data is 
Fourier transformed ( $\mathrm{F}$ engine) and then correlated ( $\mathrm{X}$ engine). The system, shown schematically below, consists of three ROACH boards running identical software with five major subsystems: the ADCs, the F engine, the inter-board data links, the $\mathrm{X}$ engine, and the server data link.

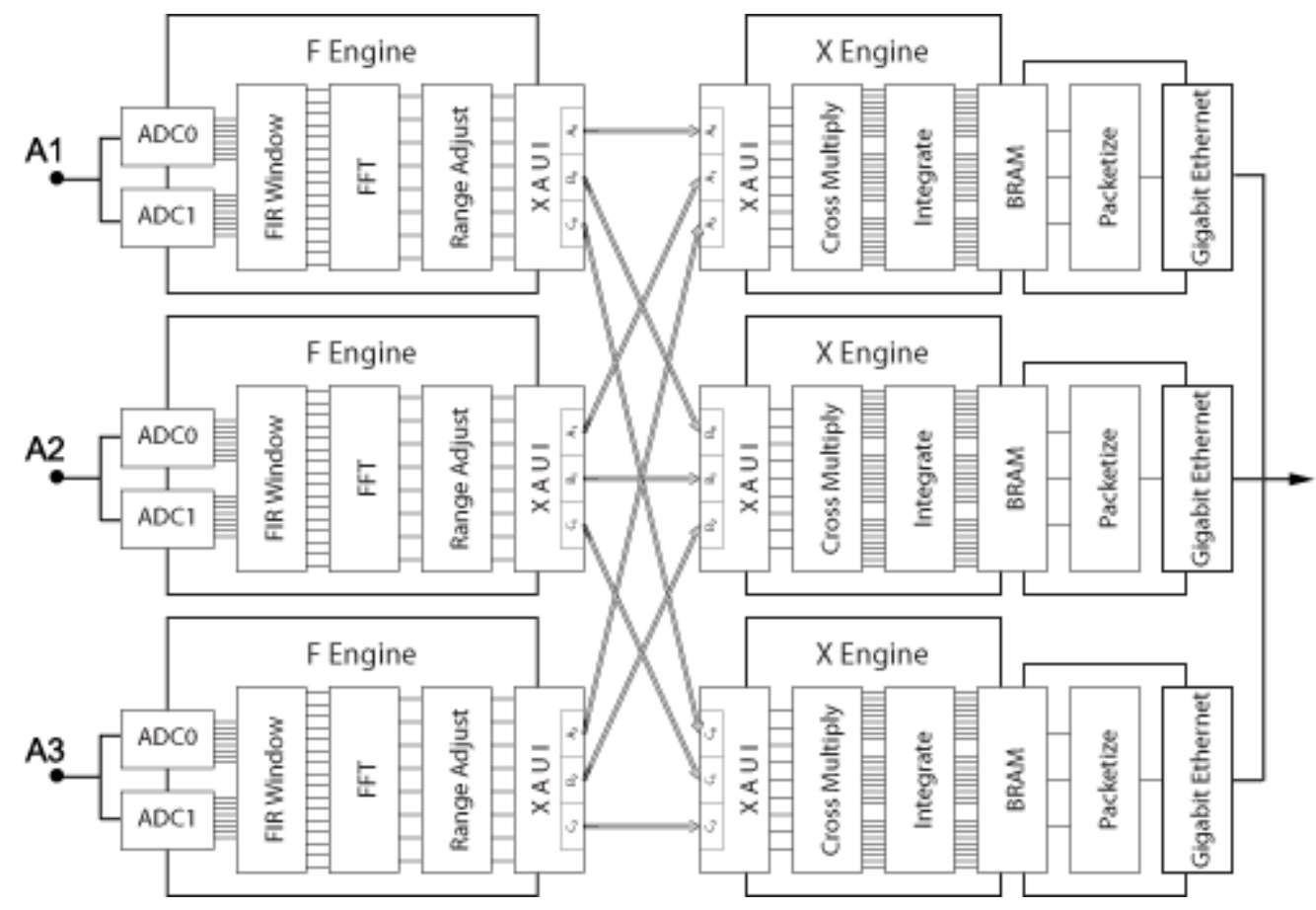

Figure 7. Schematic diagram of the correlator. Each horizontal sequence of operations takes place on one of the three ROACH boards. Signals at inputs A1, A2, A3 (see Figure 1) are digitized at 6 GSps, windowed and Fourier transformed. Subsets of the 64 spectral channels are swapped between the boards for cross-correlation every $21 \mathrm{~ns}$ over the $10 \mathrm{Gbps}$ XAUI links. Data is integrated over $1 \mathrm{~ms}$ intervals and then sent to a server.

The correlator receives signals from the three ISI telescopes after the RF delay lines at the "taps" shown in Figure 1. Each board digitizes the RF signal from a single telescope at 6 GSps (giga-samples per second) using a pair of interleaved 3 GSps, 8 bit ADCs. The F engine is a PFB (polyphase filter bank), which breaks the stream of sequential ADC samples into 16 parallel streams at $1 / 16$ the sampling rate, and then performs all signal processing at this lower data rate. These 8 bit data streams are first windowed with a Hamming function, which is implemented as a four-tap FIR filter. The output is then Fourier transformed to produce 18 bit real and 18 bit imaginary spectral components. To reduce inter-board data rates, these are each reduced to 4 bits by the "range adjust" block in the diagram. Each $F$ engine produces 64 complex spectral components every $21 \mathrm{~ns}$.

These 64 components are then split between the boards so that each one correlates all data for roughly $1 / 3$ of the spectral channels. Data is redistributed between boards via three $10 \mathrm{Gbps}$ XAUI links, as indicated in the drawing. For correlation to work, all spectral components must arrive synchronously in each $\mathrm{X}$ engine. Since XAUI is an asynchronous network protocol, each board must resynchronize the streams received from all three $\mathrm{F}$ engines before passing the data to the $\mathrm{X}$ engine. Each $\mathrm{X}$ engine performs multiplications on a channel-by-channel basis to produce autocorrelations for each telescope and cross-correlations for each baseline. The $\mathrm{X}$ engines then integrate these values for approximately $1 \mathrm{~ms}$, and they send the integrated data via ethernet to a server for storage and off-line analysis.

The new correlator is currently being tested. It recently produced the first transformed and correlated data from test signals in the laboratory, and some of this data is shown below. The system is working at $53 \%$ of the full sampling speed, and some synchronization issues during inter-board transfers have yet to be solved. The processing of the new system differs from that of the current ISI system and it is instructive to trace through the data analysis sequence. 


\subsection{Processing of spectrometer-correlator data}

The analysis of ISI data relies on a Fourier transform relationship, described by the van Cittert-Zernike theorem, between the complex correlation function of signals received at two telescopes and the brightness distribution of the source. Consider a two-dimensional source with intensity distribution $B(l, m, v) \mathrm{W} \operatorname{srr}^{-1} \mathrm{~Hz}^{-1}$, where $l$ and $m$ are direction cosines and $v$ is the radiation frequency. The measured electric field, $\varepsilon_{i}(t)$ at telescope $i$, where the index $i$ includes telescopes 1,2 and 3 , as a function of time $t$ integrated over solid angle $\Omega$ is

$$
\varepsilon_{i}(t)=\int \frac{B(l, m, v)}{R_{i}} e^{-j 2 \pi v\left(t+R_{i} / c\right)} d \Omega,
$$

where $R_{i}$ is the distance between the source and telescope $i$ and $c$ is the vacuum speed of light. The correlation function between two telescopes, for example telescopes 1 and 2 , is defined as

$$
\Gamma_{1,2}=\left\langle\varepsilon_{1} \varepsilon_{2}^{*}\right\rangle,
$$

where the angled brackets denote time-averaging. In the case of a small source, where $\sqrt{1-l^{2}-m^{2}} \sim 1$, and assuming spatial incoherence in the source, the correlation function is approximated as

$$
\Gamma_{1,2}=\int I(l, m, v) e^{-j 2 \pi(l u+m v)} d l d m
$$

where $u=x c / v$ and $v=y c / v$ are the $U V$ spatial frequencies and $I(l, m, v)=B(l, m, v)^{2} / R_{1} R_{2}$.

This correlation function is the interferometric fringe that is measured by each ISI baseline, which, when normalized, forms the complex visibility. The current ISI heterodyne detection system, as mentioned above, is designed to combine telescope IF signals so that the fringes appear as audio frequency modulations of the combined, then detected, radio frequency power. The amplitude and phase of the fringes are measured by Fourier transforming the recorded data and integrating spectral components centered at 86,107 and $193 \mathrm{~Hz}$ for the three telescope baseline pairs. In contrast, the new correlator provides separate time series for the real and imaginary parts of the 64 spectral channels, which again encode the audio frequency fringes.

The method by which fringes are measured in the new correlator is illustrated by the processing of recently acquired test data. A sinusoid with frequency $150 \mathrm{MHz}+99 \mathrm{~Hz}$ was input into ROACH board A, and an independent sinusoid with a frequency of $150 \mathrm{MHz}$ is input to ROACH boards B and C. The sampling rate was $3.2 \mathrm{GSps}$ and signal levels were adjusted to fill the 8 bits of the ADC. The left panel of Figure 8 shows 512 samples from board A. The windowing function is shown by the red dashed curve. The 512 signal values are multiplied by the window function and the result is folded to form the 128 points shown in the middle panel. The 512 point window is continuously moving with a stride of 128 points over the ADC data stream. The Fourier transform of the windowed data is shown in the right panel. In addition to the spectral feature at $150 \mathrm{MHz}$ there are small features at $1450 \mathrm{MHz}$. These "ghost" spectra appear at the Nyquist frequency- $150 \mathrm{MHz}$, and they are caused by imperfect interleaving of the ADCs. The sampling error between the even and odd ADC samples is about 0.1 of a sampling interval. This error can be corrected by adjusting delay registers on the ADCs.
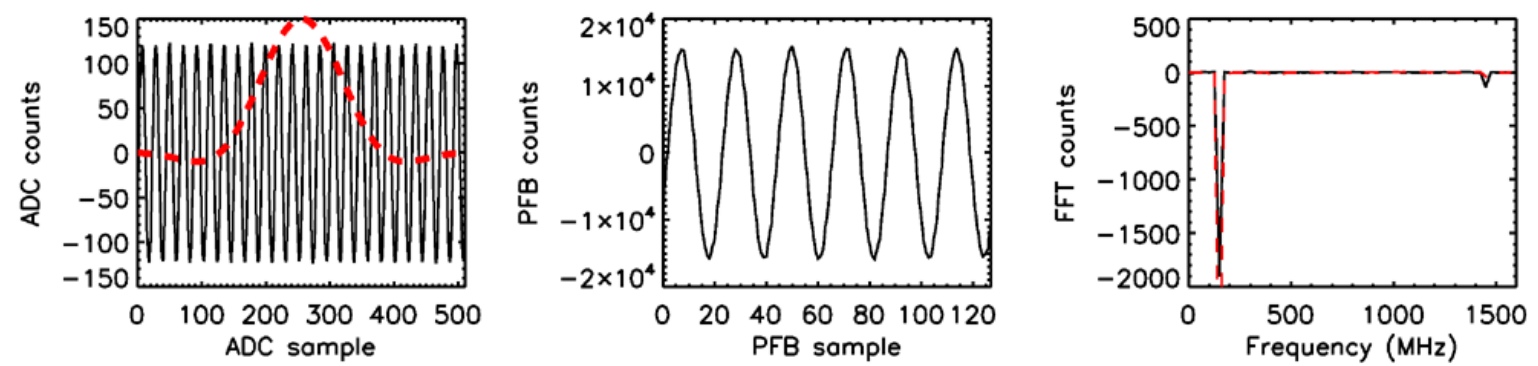

Figure 8. Left panel, the black curve shows digitized data from board A and the red dashed curve shows the window function (with maximum value unity). Middle panel, 128 data points after windowing and folding. Right panel, the FFT of the middle panel data, real (black) and imaginary (red). Small features at Nyquist-150 MHz are due to ADC sampling errors. 
The real and imaginary components of each spectral channel are then distributed among the ROACH boards for correlation. For each spectral channel, the complex Fourier components measured by one board are conjugate-multiplied with those of the other boards. For example, board A values are multiplied by the conjugates of board B values, B values are multiplied by the conjugates of $\mathrm{C}$, and $\mathrm{C}$ values by the conjugates of $\mathrm{A}$. The real and imaginary components of each conjugate multiplication are then integrated independently on $1 \mathrm{~ms}$ intervals. If the real and imaginary parts are combined before integration, then the modulation of the fringe is lost.

The left panel of Figure 9 shows 50 integrations from a 3.5 second time series. The plot shows the real (solid black line) and imaginary (red dashed line) components of the multiplication and integration of signals from boards A and B and only components from RF spectral channel 6 , or the $150 \mathrm{MHz}$ channel, are shown. The modulation in both signals with a period of approximately $10 \mathrm{~ms}$ is caused by the frequency difference of $99 \mathrm{~Hz}$ between the sinusoids sampled by boards A and B. These time series are examples of the spectrometer-correlator output. The right panel of Figure 9 shows a section of the Fourier transform of the time series for all 64 of the RF channels. The $99 \mathrm{~Hz}$ audio fringe is clearly seen in the $150 \mathrm{MHz}$ RF channel and no significant amplitudes are seen at any other audio, or RF frequencies.
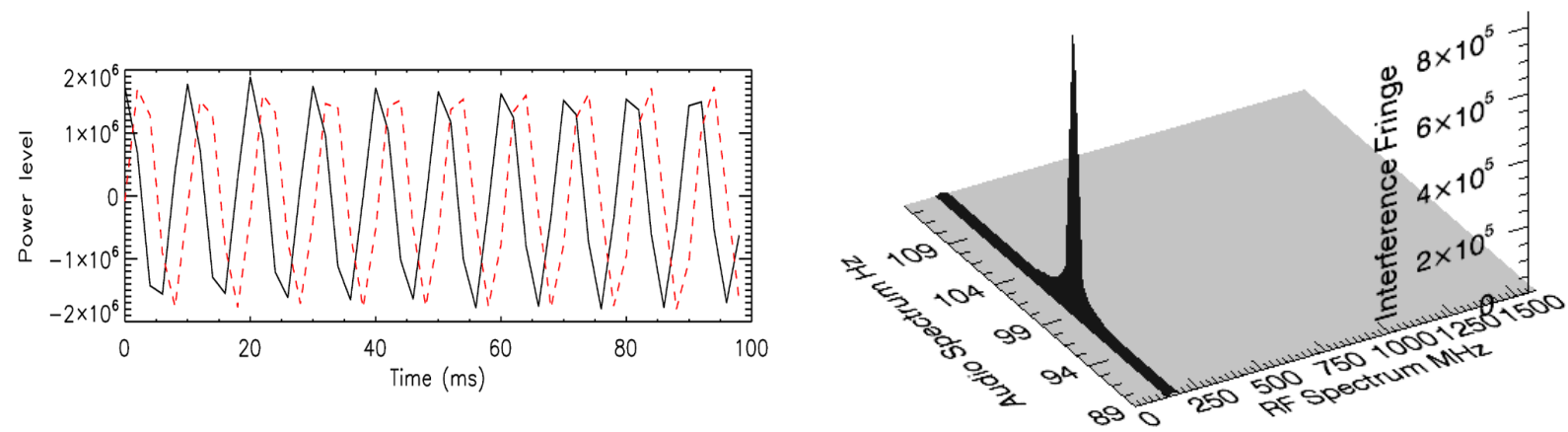

Figure 9. Left panel, the AB time series for $1 \mathrm{~ms}$ integrations for the $150 \mathrm{MHz}$ RF channel. The solid black line shows the real series and the dashed red line shows the imaginary series. A surface plot of the AB two-dimensional data set. One axis is the RF spectrum and the other is a section of the audio spectrum. For the test example, the audio fringe is indicated by the peak at $99 \mathrm{~Hz}$.

The final processing stage, not illustrated here, is the calculation of the complex visibilities. The squares of the visibility amplitudes are calculated by normalizing the squares of the fringe amplitudes by the products of the individual telescope powers. This process is carried out in each RF spectral channel. The telescope powers are measured by analyzing the autocorrelation time series for each RF spectral channel for each telescope. The visibility phase is not a well determined quantity due to atmospheric fluctuations. The closure phase, however, will be measured in a manner described by Hale et al., ${ }^{19}$ and closure phase will be obtained for each RF spectral channel.

The new correlator is designed to operate in parallel with the current ISI system. This will enable continued long term monitoring of stellar variations using the current system and it will also enable a cross-calibration of the new spectrometer-correlator. The visibilities obtained using data integrated over the 64 channel RF spectrum must be the same as those measured by the current full bandwidth ISI analog system.

\section{SUMMARY}

The ISI has been conducting mid-infrared interferometric observations of red giant and Mira stars over short and long time scales. Observations of the star W Hydrae during the year 2008 indicate that a shell of dust, near the stellar surface, has moved radially outward from the star. Recent measurements of the star Betelgeuse, have been fitted with simple image models, and in the case of a uniform disk, give a diameter of 52 mas. This measurement is larger than sizes observed about a year ago. The increase in size differs from the diminishing sizes that have been observed over the previous 16 years. The ISI is developing a new correlator system in order to obtain visibility measurements on-and-off individual molecular spectral lines. This new system uses high-speed digital signal processing of the three heterodyne IF signals. The testing of the system is underway and spectroscopic-interferometry observations will be conducted in the next year. 


\section{ACKNOWLEDGMENTS}

K. Reichl and V. Toy provided very valuable assistance with the stellar observations. J. Cobb provided computer assistance, P. Reichl revised data acquisition computer programs, and D.D.S. Hale provided overall system advice. The FPGAs were generously donated by Xilinx. We gratefully acknowledge the support of the National Science Foundation, the Gordon and Betty Moore Foundation and the Office of Naval Research.

\section{REFERENCES}

[1] Hale, D.D.S. et al., "The Berkeley Infrared Spatial Interferometer: A Heterodyne Stellar Interferometer for the MidInfrared," Ap J, 537, 998 (2000)

[2] Weiner, J., Hale, D.D.S. and Townes, C.H., "The variability of late-type stars' diameters measured using midinfrared interferometry," SPIE Proc 4838, 172, (2003)

[3] Weiner, J., Hale, D.D.S. and Townes, C.H., "Interferometry on Mira in the Mid-Infrared: Cyclic Variability of the Continuum Diameter and the Effect of Spectral Lines on Apparent Size," Ap J, 588, 1064 (2003)

[4] Wishnow, E.H., Townes, C.H., Walp, B., and Lockwood, S., "The Dust Surrounding W Hydrae," ApJ, 712, L135 (2010)

[5] Woodruff, H.C., et al., "The Keck Aperture Masking Experiment: Multiwavelength Observations of Six Mira Variables," ApJ, 673, 418 (2008)

[6] Reid, M.J. and Menten, K.M, "Imaging the Radio Photospheres of Mira Variables," ApJ, 617, 2068 (2007)

[7] Reid, M.J. and Menten, K.M., "A Subarcsecond $\mathrm{H}_{2} \mathrm{O}$ Maser Shell Surrounding a Variable Star," ApJ, 360, L51 (1990)

[8] Tatebe, K., Chandler, A.A., Wishnow, E.H., Hale, D.D.S., and Townes, C.H., "The Nonspherical Shape of Betelgeuse in the Mid-Infrared," ApJ, 670, L21 (2007)

[9] Tatebe, K, Hale, D.D.S., Wishnow, E.H. and Townes, C.H., "Observation of a Burst of High-Velocity Dust from $\alpha$ Herculis," Ap J, 658, L103 (2007)

[10] Townes, C.H., Wishnow, E.H., Hale, D.D.S., and Walp, B., "A Systemaic Change with Time in the size of Betelgeuse," ApJ, 697, L127 (2009)

[11] Chiavassa, A., Plez, B. Josselin, E., and Freytag, B. "Radiative hydrodynamics simulations of red supergiant stars: I. interpretation of interferometric observations," A\&A, 506, 1351 (2009)

[12] Perrin, G. et al. "The molecular and dusty composition of Betelgeuse's inner circumstellar envirnonment," A\&A, 474, 599 (2007)

[13] Tsuji, T., "Infrared Spectra and Visibilities as Probes of the Outer Atmospheres of Red Giant Stars," ApJ, 645, 1448 (2006)

[14] Ohnaka, K. et al., "Spatially resolving the inhomogeneous structure of the dynamical atmosphere of Betelgeuse with VLT/AMBER," A\&A, 503, 183 (2009)

[15] Monnier, J.D., et al. "Mid-Infrared Interferometry on Spectral Lines. III. Ammonia and Silane around IRC +10216 and VY Canis Majoris," Ap J, 543, 868 (2000)

[16] Lacy, et al. "TEXES: A Sensitive High-Resolution Grating Spectrograph for the Mid-Infrared," PASP, 114, 153 (2002)

[17] Rothman, L.S., et al. "The HITRAN Molecular Spectroscopy Database and HAWKS 1996 Edition,” JQSRT, 60, 665 (1998)

[18] Parsons, A., et al. "A Scalable Correlator Architecture Based on Modular FPGA Hardware, Reuseable Gateware, and Data Packetization," PASP, 120, 1207 (2008)

[19] Hale, D.D.S., et al. "Techniques for Measuring Phase Closure at 11 Microns," SPIE Proc 4838, 387, (2003) 